\title{
A survey of stack-sorting disciplines
}

\author{
Miklós Bóna \\ Department of Mathematics, University of Florida \\ Gainesville FL 32611-8105 \\ bona@math.ufl.edu*
}

Submitted: May 19, 2003; Accepted: Jun 18, 2003; Published: Jul 27, 2003

MR Subject Classifications: 05A15, 05A16

\begin{abstract}
We review the various ways that stacks, their variations and their combinations, have been used as sorting devices. In particular, we show that they have been a key motivator for the study of permutation patterns. We also show that they have connections to other areas in combinatorics such as Young tableau, planar graph theory, and simplicial complexes.
\end{abstract}

\section{Introduction}

The stack sorting problem introduced by Knuth [29] in the 1960's was a founding inspiration in the study of permutation patterns. Simultaneously it introduced the notion of pattern containment, defining a class of permutations by a forbidden set, and the enumeration of permutations in such classes. Soon afterwards various generalizations by Tarjan [36], Pratt [33], and Even and Itai [24] were studied and these authors posed questions about permutation patterns which even today cannot be easily answered. But, from around 1973 through to 1992 when Herbert Wilf delivered an influential address to the SIAM meeting on Discrete Mathematics, these questions lay almost untouched. The 1990's and the new millenium saw a renaissance of permutation pattern research and stack sorting problems returned as one of its main drivers. In this survey we shall review both the early work and more recent work on stack sorting. We shall see that it touches on many areas of combinatorics and remains a fascinating source of open problems.

\footnotetext{
*Supported by a Young Investigator Grant of the National Security Agency. The paper was written during a one-month stay of the author at LABRI, at the University of Bordeaux I, France.
} 
A stack is a last-in, first-out linear sequence accessed at one end called the top. Items are added and removed from the top end by push and pop operations. In its simplest form a stack is used to rearrange a permutation $p=p_{1}, p_{2}, \ldots, p_{n}$ as follows. The elements of $p$ are pushed onto an initially empty stack and an output permutation is formed by popping elements from the stack. The output permutation obviously depends on how the push and pop operations are interleaved.

In the simplest stack sorting problem one wishes the output permutation to be $1,2, \ldots, n$ (so that one has sorted the input). A necessary but not sufficient condition for this to be possible is that the stack values should always be increasing (read from the top end). Enforcing this condition leads to a "greedy algorithm" that only pops a stack symbol if pushing the next input symbol would have violated the increasing property of the stack. We let $s(p)$ be the output resulting from this greedy algorithm.

Example 1.1 Let $p=2413$. Then the stages of the greedy sorting procedure are shown in Figure 1.

\begin{tabular}{|r|c|c|}
\hline INPUT & STACK & оUTPUT \\
\hline 2413 & & \\
\hline 413 & 2 & \\
\hline 413 & & 2 \\
\hline 13 & 4 & 2 \\
\hline 3 & 1 & 2 \\
\hline 3 & 4 & 21 \\
\hline & 3 & 21 \\
\hline & 4 & 213 \\
\hline & & 2134 \\
\hline
\end{tabular}

Figure 1: Sorting 2413

There is an alternative, recursive definition of the function $s(p)$. The following Lemma makes this evident.

Lemma 1.2 Let $p=\operatorname{Ln} R$ be an n-permutation, where $L$ denotes the string on the left of the entry $n$, and $R$ denotes the string on the right of the entry $n$. Then we have

$$
s(p)=s(L) s(R) n .
$$

Proof: The entry $n$ can enter the stack only when it is empty, that is, when $L$ has passed through the stack. Once $n$ is in the stack, it will stay there until the end, so $R$ will past through the stack after $L$. $\diamond$

The following result of Knuth [29] originated the study of stack sorting. 
Proposition 1.3 A permutation $p$ can be sorted by a stack if and only if $s(p)$ is the identity permutation and this happens if and only if $p$ avoids the pattern 231. The number of such sortable permutations of length $n$ is $C_{n}=\left(\begin{array}{c}2 n \\ n\end{array}\right) /(n+1)$.

The remainder of this paper surveys various ways in which Proposition 1.3 has been generalized and refined. The generalizations have been of two kinds. In the first (discussed in section 2) we replace the stack by a linear sequence with more complex accessing rules and in the second (section 3 ) we use several stacks connected together in various ways. For all of these generalizations there is an enumeration question: how many $n$-permutations can be sorted by the system. For most of them the set of sortable permutations is closed under forming subpermutations and so there is the question of finding the minimal set of unsortable permutations; the set of sortable permutations is then characterized as those permutations which avoid this minimal set. As we shall see, there is one problem where the sortable set is not closed under subpermutations and that gives rise to a number of rather different considerations. The main refinement we consider is enumeration by length and number of descents (section 4). Here detailed information is rather patchy but for one class of stack-sortable permutations at least there are some encouraging beginnings and intriguing connections with planar graph theory.

\section{Variations on a single stack}

The first generalizations of the ordinary stack appeared a few pages later in [29] than the proof of Proposition 1.3. An input-restricted deque is like a stack in that it has a push operation but the pop operation can remove an element from either end of the sequence. By an ingenious argument that subsequently grew into what we nowadays call the kernel method Knuth proved

Proposition 2.1 A permutation can be sorted by an input-restricted deque if and only if it has no subpermutation of the form 4231 or 3241 . The numbers of sortable n-permutations are given by the Schröder numbers $s_{n}$ whose generating function is

$$
\sum_{n=0}^{\infty} s_{n} x^{n}=\frac{3-x-\sqrt{1-6 x+x^{2}}}{2}
$$

Knuth also posed the problem of sorting using a (general) deque where one can push and pop at either end. It was proved by Pratt [33] that the deque sortable permutations are characterized by avoiding a certain infinite set $A$ of permutations. The set $A$ was the first published example of an infinite antichain in the pattern containment order. However, the enumeration question for deques remains completely unsolved.

Of course deques (input-restricted or not) are more powerful than stacks in that they sort a wider collection of permutations than stacks. In [8] Avis and Newborn defined a weaker 
structure that they called a pop-stack (of which more in section 3). The permutations that a pop-stack can sort are exactly the layered permutations of [10]; their characterizing unsortable set is $\{231,312\}$ and they are enumerated by the function $2^{n-1}$.

A family of more general stack-like structures was defined by Atkinson [4]. In an $(r, s)$ stack one is allowed to push into any of the first $r$ positions and pop from any of the $s$ positions at the top end of the stack. Of course, an ordinary stack corresponds to $r=s=1$. For all of the cases $r=1, s=1$, and $r=s=2$ the characterizing unsortable set is known and is finite. The $(r, 1)$-stack-sortable permutations (and the $(1, s)$-stack sortable permutations) have been enumerated in that their ordinary generating functions are known. It happens that the $(2,1)$ - stack sortable permutations are enumerated by the Schöder numbers although there is no symmetry that maps the problem directly to an input-restricted deque problem.

Another family of stack-like structures was defined by Albert and Atkinson in [1]. An $(r, s)$ fork-stack has a push operation that can transfer a contiguous block of at most $r$ symbols from the input to the stack and a pop operation that can transfer a block of at most $s$ symbols from the stack to the output. For all values of $r$ and $s$ it is known that there are only finitely many minimal unsortable permutations (even if either parameter is infinite). Very strong conditions are known on the generating functions in most cases. Except for the case $r=s=\infty$ the generating function is algebraic and it is known explicitly when either $r=1$ or $s=1$ or $r=s=2$.

Finally in this section we note that all these problems have variations where one restricts the size of the stack structure. As part of a much more general investigation of bounded structures Atkinson, Livesey, and Tulley [3] solved the minimal unsortable permutation problem and the enumeration problem for ordinary stacks that are not allowed to contain more than some fixed number of symbols. It turns out that the corresponding generating functions are rational (unlike the Catalan generating function associated with unbounded stacks).

\section{Systems of stacks}

Again it was Knuth in [29] who first posed questions about systems of stacks (using the language of railway sidings). His ideas were taken up by Tarjan [36] who defined a very general model. In Tarjan's model one has a graph whose nodes are stacks (or queues). If two stacks are connected by an edge from $A$ to $B$ then items popped from stack $A$ are pushed onto stack $B$. In this generality virtually nothing can be proved but there are two special cases that have attracted several researchers over many years.

The first special case is of stacks $S_{1}, S_{2}, \ldots, S_{t}$ in parallel. At any point in the sorting process we may push the next input symbol onto one of the stacks or we may pop one of the stacks and thereby create another symbol of the output permutation. Nothing is known about the enumeration of the sortable permutations (except, of course, if $t=1$ ), 
although the methods of [3] apply if there is a bound on the size of the stacks. However, for $t=1,2,3$, there is at least an efficient algorithm (complexity $O(n \log n)$ ) to decide whether a permutation is sortable. The algorithm stems from a reduction by Even and Itai [24] to a graph-coloring problem and a solution to this coloring problem by Unger [38]. It is also known [33] that the set of minimal unsortable permutations is infinite if $t \geq 2$ (and this remains true even for two stacks of size 2 in parallel [3]). The situation is simpler for a system of pop-stacks in parallel; here there is always a finite number only of minimal unsortable permutations (and the enumeration problem has been solved when $t=2[5])$.

Rather more work has been done on stacks $S_{1}, S_{2}, \ldots, S_{t}$ in series. Here we seek to sort a permutation by pushing its symbols onto $S_{1}$, popping them off $S_{1}$ and on to $S_{2}$, transferring them from $S_{2}$ and pushing them on to $S_{3}$, etc., until they emerge from $S_{t}$ to become new output symbols.

If no further conditions apply we call this sorting model General stacks in series. The earliest result was proved in [29]: every $n$-permutation can be sorted by $\log _{2} n$ stacks in series. It is not difficult to see that the sortable permutations are exactly those that can be expressed as a composition $\pi_{1} \pi_{2} \ldots \pi_{t}$ where each factor is a permutation that can be sorted by a single stack. Therefore the number of permutations that $t$ stacks in series can sort is at most $O\left(4^{t n}\right)$. Unfortunately, the same permutation can be expressed as a composition in many different ways so this bound is probably not tight.

There is another method for finding upper bounds on the number of sortable permutations that was first suggested in [2] and later, in a different context, in [27]. We describe the process whereby the permutation $p$ is sorted using words from an alphabet $A_{1}, A_{2}, \ldots A_{t+1}$. The letter $A_{i}$ denotes the operation of moving a symbol from the $(i-1)$ th stack to the $i$ th stack (where $i=1$ and $i=t+1$ correspond to moves onto the first stack from the input and moves from the last stack to the output). Since every symbol undergoes $t+1$ moves these words have length $n(t+1)$ and each letter occurs exactly $n$ times. Not every word of this form is possible since a stack can only be popped if it is non-empty and, in fact, these stack words are in one-to-one correspondence with $(t+1) \times n$ rectangular standard Young tableau so they may be enumerated by the hook formula. This tells us that the number of stack words is

$$
\frac{(t n+n) ! 2 ! 3 ! \cdots t !}{n !(n+1) ! \cdots(n+t) !}
$$

Example 3.1 Let $p=132$. Then the stages of our sorting procedure are shown on Figure 2. This shows that the stack word associated to $p$ is $A_{1} A_{1} A_{1} A_{1} A_{3} A_{1} A_{3} A_{2} A_{3}$.

In its raw form this method gives worse upper bounds than the one above but in at least one case [6] it has been refined to give an accurate result. In general, however, there are very many operation sequences that sort the same permutation and no way of choosing a canonical sequence of operations is known. In particular, even for $t=2$ no efficient algorithm is known to decide whether a permutation is sortable and it is possible that this decision problem is NP-complete. 


\begin{tabular}{|c|c|c|c|}
\hline INPUT & FIRST STACK & SECOND STACK & OUTPUT \\
\hline 132 & & & \\
\hline 32 & 1 & & \\
\hline 32 & 3 & 1 & \\
\hline 2 & 23 & 1 & \\
\hline & 23 & & 1 \\
\hline & 3 & 2 & 12 \\
\hline & 3 & & 123 \\
\hline & & 3 & \\
\hline & & & 12 \\
\hline
\end{tabular}

Figure 2: Sorting 132 with two serial stacks

The minimal unsortable permutations also present great difficulties. For $t=2$ Murphy [32] has proved that there an infinite number of them but the actual set is unknown (its smallest permutations are of length 7).

To make further progress on systems of stacks connected in series we have to impose further conditions. Atkinson, Murphy, and Ruskuc [6] considered stacks in series where each stack is constrained to have increasing values from the top. We shall call this model Increasing stacks in series. For the case $t=2$ it is known that there are an infinite number of characterizing minimal unsortable permutations and the enumeration problem has been solved. Surprisingly, the number of sortable $n$-permutations is equal to the number that avoid the permutation 1342 (enumerated in [9]). Both [6] and [9] rely on the enumeration of a combinatorial structure called a $\beta(0,1)$-tree.

There is a widely studied and even more restricted model that we shall call Greedy increasing stacks in series. In this model there is a rule that determines which push or pop operation must be carried out at each step. We examine the stacks in the order $S_{1}, S_{2}, \ldots, S_{t}$ and locate the first stack on which a push can be applied (and would keep the stack in increasing order); if there is no such stack then stack $S_{t}$ is popped to produce a new output symbol. It is easy to verify that a permutation $p$ is sortable in this model if and only if $s^{t}(p)=12 \cdots n$. It is then clear by Lemma 1.2 that all $n$-permutations are $(n-1)$-stack sortable in this model. The greedy increasing model was introduced by Julian West [39] who defined the sortable permutations by the equation $s^{t}(p)=12 \cdots n$. At that time the problem had not been recognized as having a wider context and so, in the literature, the sortable permutations are often referred to simply as $t$-stack sortable permutations but, for clarity, we shall call them West- $t$-sortable permutations. We let $W_{t}(n)$ denote the number of West-t-sortable $n$-permutations.

These classes of permutations differ from all the above classes in not being closed under subpermutations and this is a major reason why they are hard to handle. For example 35241 is West-2-sortable but its subpermutation 3241 is not. 
West [39] conjectured the following theorem.

Theorem 3.2 For all n, we have

$$
W_{2}(n)=\frac{2(3 n) !}{(n+1) !(2 n+1) !} .
$$

This conjecture was open for five years. Then it was solved by D. Zeilberger [40], who used a computer to find the solution to a degree- 9 functional equation. Two other proofs $[22,26]$ have been found later; both show fairly complicated bijections between the set of 2-stack sortable permutations of length $n$ and nonseparable rooted planar maps on $n+1$ edges. The latter have been enumerated by Tutte in 1963 [37]. Finally, a simpler (but not simple) proof has been found [21] that constructs a bijection between two-stack sortable permutations, and a certain class of labeled trees called $\beta(1,0)$-trees. As their name suggests $\beta(1,0)$-trees are similar to the $\beta(0,1)$-trees that play an important role in the enumeration of sortable permutations in the increasing stacks in series model. It would be interesting to have a unified treatment of these enumerations.

There are no exact formulae known for $W_{t}(n)$ if $t>2$. Good upper bounds are not known either. The best estimate we can get uses the following Lemma of West [39], and a recent result of West and Stankova [34].

Lemma 3.3 If a permutation $p$ contains $q_{k}=234 \cdots k 1$ as a pattern, then $p$ is not West- $(k-2)$-stack sortable.

Proof: Induction on $k$. If $p$ contains $q_{k}$, then $s(p)$ contains $q_{k-1}$, and our claim follows. $\diamond$

Theorem 3.4 For all $n$ and $t$, we have $W_{t}(n)<(t+1)^{2 n}$.

Proof: By Lemma 3.3, all West- $t$-stack sortable permutations have to avoid $23 \cdots(t+2) 1$. We know [34] that the number of $n$-permutations avoiding $234 \cdots k 1$ is less than $(t+1)^{2 n}$, so the statement follows. $\diamond$

This bound is not sharp, unless $t=1$. Even for $t=2$, the sequence $\sqrt[n]{W_{2}(n)}$ converges to 6.75 by the formula given in Theorem 3.2, which is significantly less than the $9^{n}$ that we obtain using Theorem 3.4.

This leads us to an interesting area of research. While an exact formula is known for $W_{2}(t)$, none of the several known proofs shows in a clear way why $W_{2}(t)<\left(\begin{array}{c}3 n \\ n\end{array}\right)$. That is, it would be revealing to find an injection from the set of 2 -stack sortable $n$-permutations into that of $n$-element subsets of a $3 n$-element set. Such an injection could possibly provide us with insight for cases of larger $t$. It has to be said, however, that while for $t=1$ and 
$t=2$, the value of $W_{t}(n)$ was given by $\left(\begin{array}{c}(t+1) n \\ n\end{array}\right) / p_{t}(n)$, where $p_{t}(n)$ was a polynomial, numerical evidence computed by West in [39] did not support this trend for $t=3$. It is an intriguing question to see why, and whether the numbers $W_{3}(t)$ are smaller or larger than $\left(\begin{array}{c}4 n \\ n\end{array}\right)$ divided by some polynomial.

The hook formula (equation 1) hints that the expression $\left(\begin{array}{c}(t+1) n \\ n\end{array}\right)$ might indeed be relevant. For greedy increasing stacks in series every sortable permutation is associated with a unique stack word. But the difficulty now is that not every stack word corresponds to a greedy stack sorting process. While it is not difficult to find necessary conditions for stack words coming from $t$-stack sortable permutations, it is much more difficult to find sufficient conditions. This is because for that, we would need a characterization of the permutations that can be the image of $s^{t}$. The only results in this direction are due to M. Bousquet-Melou [16], are recursive, and are for the special case of $t=1$.

Finally, we mention a classic result originally due to Kreweras [30] that at first sight looks completely unrelated.

Theorem 3.5 The number of lattice paths starting at $(0,0)$, ending in $(i, 0)$, and using $3 n+2 i$ steps, each of which is equal to either $(1,1)$, or $(0,-1)$, or $(-1,0)$, and never leaving the first quadrant is

$$
\frac{4(2 i+1)}{(n+i+1)(2 n+2 i+1)}\left(\begin{array}{c}
2 i \\
i
\end{array}\right)\left(\begin{array}{c}
3 n+2 i \\
n
\end{array}\right) .
$$

See [17] for more information about this result. If we set $i=0$, then we get that the number of such lattice paths that end in $(0,0)$ in $3 n$ steps is precisely $4^{n} W_{2}(n)$. We do not know of a direct proof of this fact.

For all the series models above no precise enumeration results are known with $t>2$ but we can conclude this section on a more positive note. If we have an unbounded number of pop-stacks in series (in practice, any number more than $n$ ) then the number of sortable permutations is, once again, the $n$th Schröder number. Also, the minimal unsortable permutations are 2413 and 3142. These facts may be deduced from [8, 14]. It is also known [7] what the enumeration function is for any fixed number of pop-stacks in series.

\section{Enumeration of West $t$-stack-sortable permutations by ascents}

In this section we are concerned solely with West $t$-stack-sortable permutations and for brevity we call these simply $t$-stack-sortable permutations. We shall review what is known about the numbers $W_{t}(n, k)$ where $W_{t}(n, k)$ is the number of $t$-stack-sortable $n$-permutations with $k$ ascents. We propose to fix $n$ and $t$, and investigate the finite sequence $\left(W_{t}(n, k)\right)_{0 \leq k \leq n-1}$. 
To this end we recall a number of definitions. A sequence of elements of $\mathbf{R}$ (the real numbers) $\left(a_{k}\right)_{0 \leq k \leq n}$ is unimodal if there is an index $m$ such that $a_{0} \leq a_{1} \leq \ldots \leq a_{m} \geq$ $a_{m+1} \geq \ldots \geq a_{n}$.

A related concept is that of log-concavity. The sequence $\left(a_{k}\right)_{0 \leq k \leq n}$ is log-concave if $a_{k-1} a_{k+1} \leq a_{k}^{2}$ for all $k$. Such sequences abound in algebra, combinatorics, and geometry. Stanley's article [35] gives a good survey. It is easy to prove that if the numbers $a_{k}$ are positive, and the sequence $\left(a_{k}\right)_{0 \leq k \leq n}$ is log-concave, then it is unimodal.

An even stronger property for a sequence $\left(a_{k}\right)_{0 \leq k \leq n}$ is when its generating function $\sum_{k=0}^{n} a_{k} x^{k}$ has real roots only. Another classic result is that if the numbers $a_{k}$ are positive, and their generating function $\sum_{k=0}^{n} a_{k} x^{k}$ has real roots only, then $\left(a_{k}\right)_{0 \leq k \leq n}$ is log-concave, and therefore, unimodal.

We would like to study symmetry, unimodality, log-concavity, and real zeros properties of these sequences and polynomials in the case that $a_{k}=W_{t}(n, k)$.

Three special instances of these problems are completely solved.

- If $t=n-1$, then all permutations are $t$-stack sortable, so our enumeration is reduced to that of enumerating permutations with a given number of descents. Therefore, $W_{t}(n, k)=A(n, k+1)$, that is, $W_{n, t}(x)=A_{n}(x)$, in other words, we get the Eulerian polynomials. Therefore, [25], in this case our sequence is symmetric, and our polynomial $W_{n-1}(n, x)$ has real zeros only.

- If $t=1$, then the numbers $W_{1}(n, k)$ will be the famous Narayana numbers, that is

$$
W_{1}(n, k)=\frac{1}{n}\left(\begin{array}{l}
n \\
k
\end{array}\right)\left(\begin{array}{c}
n \\
k+1
\end{array}\right)
$$

In particular, the sequence $\left\{W_{1}(n, k)\right\}, 0 \leq k \leq n-1$ is symmetric, and logconcave. It is far from obvious, but true, that the generating function $W_{n, 1}(x)$ of these numbers has real roots only [20]. A simpler proof for this fact was recently found by P. Branden [18].

- If $t=2$, then determining the numbers $W_{2}(n, k)$ is much more difficult. Constructing a bijection with nonseparable planar maps, it can be shown [31] that

$$
W_{2}(n, k)=\frac{(n+k) !(2 n-k-1) !}{(k+1) !(n-k) !(2 k+1) !(2 n-2 k-1) !} .
$$

In particular, we get again that for any fixed $n$, the sequence $\left\{W_{2}(n, k)\right\}, 0 \leq k \leq$ $n-1$ is symmetric and log-concave. Also, Branden [18] has proved that $W_{n, 2}(x)$ has real zeros only.

We mention that if $t=n-2$, then our permutations are all $n$-permutations that do not end in the string $n 1$. Therefore, symmetry and log-concavity follows easily from the 
special case of $t=n-1$ (all permutations), and the real zeros property is (probably) not difficult to prove.

The present author has proved the following general theorem, settling the two weaker questions for all $t$.

Theorem 4.1 [11] For any fixed $t$ and $n$, the sequence $\left\{W_{t}(n, k)\right\}, 0 \leq k \leq n-1$ is symmetric and unimodal.

The key element of the proof is a representation of permutations by trees. If $p$ is an $n$-permutation, we associate a rooted plane tree $T(p)$ to $p$ as follows.

The root of $T(p)$ is a vertex labeled $n$, the largest entry of $p$. If $a$ is the largest entry of $p$ on the left of $n$, and $b$ is the largest entry of $p$ on the right of $n$, then the root will have two children, the left one will be labeled $a$, and the right one labeled $b$. If $n$ was the first (resp. last) entry of $p$, then the root will have only one child, and that will be a left (resp. right) child, and it will necessarily be labeled $n-1$ as $n-1$ must be the largest of all remaining elements.

Define the rest of $T(p)$ recursively, by taking $T\left(p^{\prime}\right)$ and $T\left(p^{\prime \prime}\right)$, where $p^{\prime}$ and $p^{\prime \prime}$ are the substrings of $p$ on the two sides of $n$, and affixing them to $a$ and $b$. We then call $T(p)$ the decreasing binary tree of $p$.

Example 4.2 If $p=263498175$, then $T(p)$ is the tree shown below..

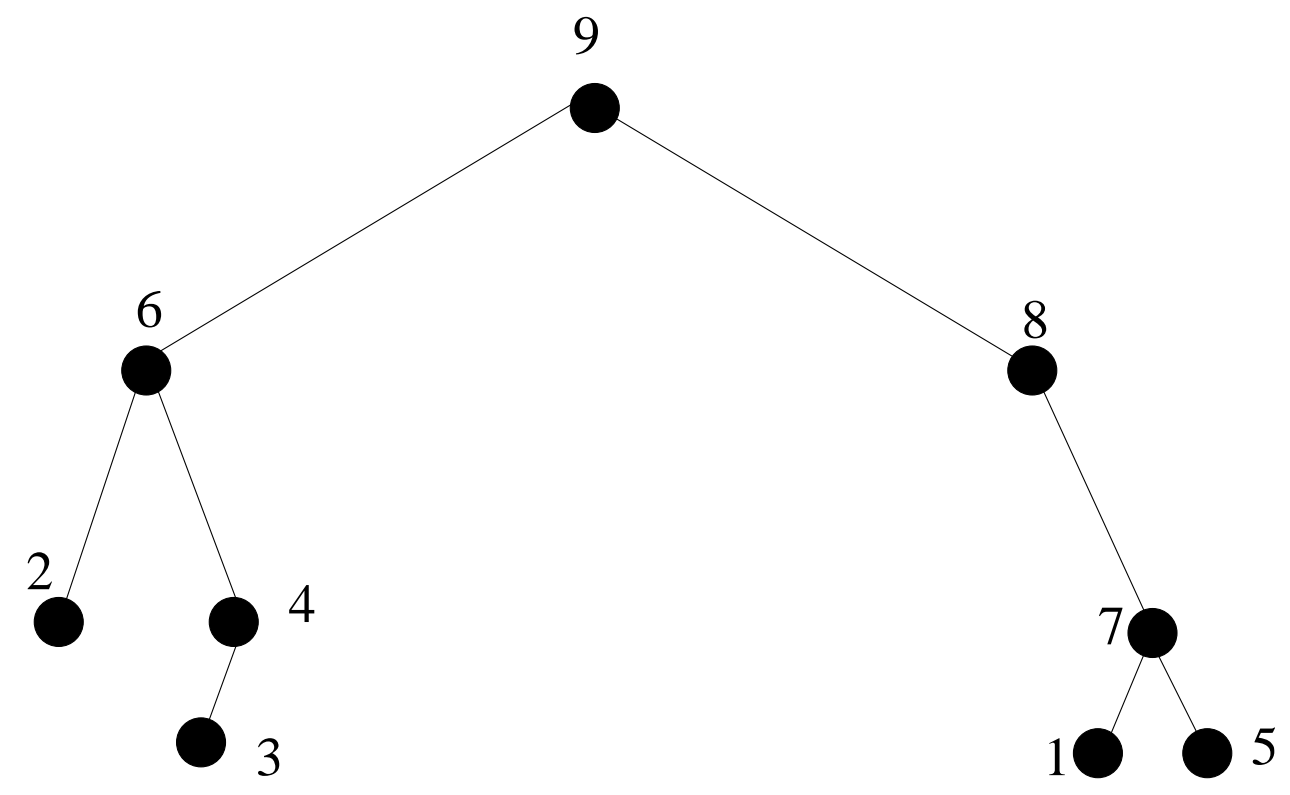

Figure 3: The decreasing binary tree of $p=263498175$ 
Proposition 4.3 Let $p$ be any n-permutation. If we read the decreasing binary tree $T(p)$ of $p$ in postorder, we obtain $s(p)$.

Note that if $p$ had $k$ ascents, then $T(p)$ has $k$ left edges. Then the symmetry of our sequence, that is, the equality $W_{t}(n, k)=W_{t}(n, n-1-k)$ is proved by the following duality map $f$.

For any decreasing binary tree $T$, leave the children of nodes with two children unchanged. However if a node has only a left child, turn that child into a right child, and if a node had only a right child, turn it into a right child.

This map $f$ is obviously an involution on the set of all binary decreasing trees on $n$ vertices. It does not change the postorder reading of the trees, so the permutations corresponding to $T$ and $f(T)$ have the same image under the stack-sorting operation. Therefore, $f$ preserves the $t$-stack sortable property while turning descents into ascents. See [11] for examples of this duality map, and see [13] for the proof of unimodality that uses the duality map $f$, and the reflection principle.

It is quite rare in combinatorics that for a sequence unimodality is easier to prove by direct combinatorial means than log-concavity. Nevertheless, it does seem to be the case here.

To state the next result we recall a definition from combinatorial geometry. A simplicial complex is a collection of sets $\Delta$ with the property that if $E \in \Delta$, and $F \subseteq E$, then $F \in \Delta$. The sets that belong to the collection $\Delta$ are called the faces of $\Delta$. If $S \in \Delta$ has $i$ elements, then we call $S$ an $(i-1)$-dimensional face. The dimension of $\Delta$ is, by definition, the dimension of its maximal faces.

Recently, V. Gasharov constructively proved the following interesting result [25] that has been proved before by F. Brenti [19] by other means, and discussed as a special case of a more general setup (Coxeter groups, instead of just the symmetric group), in [23].

Theorem 4.4 There exists a simplicial complex whose $(k-1)$-dimensional faces correspond to n-permutations with $k$ descents.

The constructive proof of Gasharov raises the following question. Is it true that for any fixed $n$, there is a simplicial complex whose $(k-1)$-dimensional faces correspond to $t$-stack sortable $n$-permutations with $k$ descents (or ascents)?

1. The case of $t=1$ is not difficult. We will show that there is a simplicial complex $S$ whose $(k-1)$-dimensional faces correspond to stack sortable permutations with $k$ ascents. It is well-known that 231-avoiding, that is, stack-sortable, $n$-permutations are in bijection with northeastern lattice paths from $(0,0)$ to $(n, n)$ that never go above the main diagonal. It is also well-known that this bijection turns the ascents of the permutations into NE turns of lattice paths. Therefore, all we have to do is to 


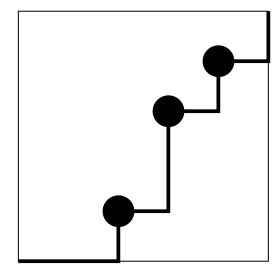

$\mathrm{L}$

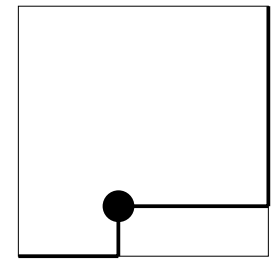

$\mathrm{L}_{1}$

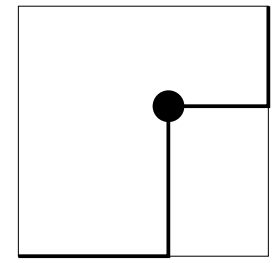

$\mathrm{L}_{2}$

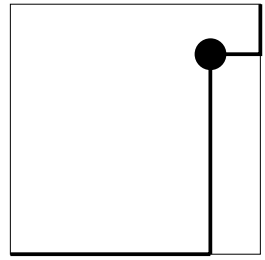

$\mathrm{L}_{3}$

Figure 4: From one lattice path to three.

decompose a northeastern lattice path with $k$ NE turns into a $k$-tuple of northeastern lattice paths with one NE turn each. This can be done as shown in Figure 4.

2. When $t=2$, then we have to resort to a much deeper representation theorem of our permutations. This representation was given in [21] and it creates a bijection between 2 -stack sortable permutations and $\beta(1,0)$-trees.

Definition 4.5 [21] [31] A rooted plane tree with positive integer labels l(v) on each of its nodes $v$ is called a $\beta(1,0)$-tree if it satisfies the following conditions:

- if $v$ is a leaf, then $l(v)=1$,

- if $v$ is the root and $v_{1}, v_{2}, \cdots, v_{k}$ are its children, then $l(v)=\sum_{i=1}^{k} l\left(v_{k}\right)$,

- if $v$ is an internal node (that is, not the root, and not a leaf), and $v_{1}, v_{2}, \cdots, v_{k}$ are its children, then $l(v) \leq \sum_{i=1}^{k} l\left(v_{k}\right)$.

See Figure 5 for an example.

The relevance of $\beta(1,0)$-trees to our problem is revealed by the following Theorem, which is quite difficult to prove.

Theorem 4.6 [21] [31] There exists a bijection $b$ from the set of all $\beta(1,0)$-trees on $n+1$ nodes onto that of all 2-stack sortable $n$-permutations so that if a $\beta(1,0)$-tree $T$ has $k$ internal nodes, then $b(T)$ has $k$ ascents.

The decomposition takes a $\beta(1,0)$-tree and splits its internal nodes into leaves. See [12] for details.

It would be interesting to see similar decompositions for $t>2$. 


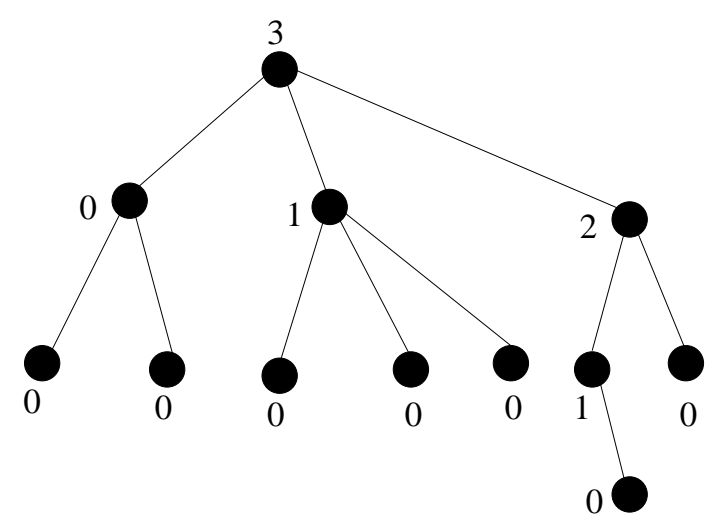

Figure 5: A $\beta(1,0)$-tree .

\section{References}

[1] M. H. Albert, M. D. Atkinson, Sorting with a forklift, Electronic J. Combinatorics, 9 (2), (2003), Paper R9.

[2] M. D. Atkinson, Sorting permutations with networks of stacks, Technical Report TR-210 (1992), School of Computer Science, Carleton University.

[3] M. D. Atkinson, M. J. Livesey, D. Tulley, Permutations generated by token passing in graphs, Theoretical Computer Science, 178 (1997), 103-118.

[4] M. D. Atkinson, Generalised stack permutations, Combinatorics, Probability and Computing, 7 (1998), 239-246.

[5] M. D. Atkinson, J.-R. Sack, Sorting with parallel pop-stacks, Information Processing Letters, 70 (1999), 63-67.

[6] M. D. Atkinson, M. M. Murphy, N. Ruškuc, Sorting with two ordered stacks in series, Theoretical Computer Science, 289 (2002), 205-223.

[7] M. D. Atkinson, T. Stitt, Restricted permutations and the wreath product, Discrete Math., 259 (2002), 19-36.

[8] D. Avis, M. Newborn, On pop-stacks in series, Utilitas Mathematica, 19 (1981), 129-140.

[9] M. Bóna, Exact enumeration of 1342-avoiding permutations: a close link with labeled trees and planar graphs, J. Combin. Theory Ser. A, 80 (1997), 257-272.

[10] M. Bóna, The solution of a conjecture of Stanley and Wilf for all layered patterns. J. Combin. Theory Ser. A, 85 (1999), 96-104. 
[11] M. Bóna, Symmetry and Unimodality in Stack sortable permutations, J. Combin. Theory Ser. A, 98, (2002) no. 1, 201-209.

[12] M. Bóna, A simplicial complex of 2-stack sortable permutations. Advances in Applied Mathematics, 29 (2002), 499-508.

[13] M. Bóna, Corrigendum to Symmetry and Unimodality in Stack sortable permutations, J. Combin. Theory Ser. A, 99 (2002) no.1, 191-194.

[14] P. Bose, J. F. Buss, A. Lubiw, Pattern matching for permutations, Inform. Process. Lett., 65 (1998) 277-283.

[15] M. Bousquet-Melou, Multi-statistic enumeration of two-stack sortable permutations, Electronic J. Combinatorics, 5 (1998), Paper R21.

[16] M. Bousquet-Melou, Sorted and/or sortable permutations, Discrete Math., 225 (2000), no. 1-3, 25-50.

[17] M. Bousquet-Melou, Counting Walks in the Quarter plane, in Mathematics and Computer Science: Algorithms, trees, combinatorics and probabilities, Trends in Mathematics, Birkhauser, 2002, pp. 49-67.

[18] P. Branden, The generating function of two-stack sortable permutations by descents is real-rooted. preprint.

[19] F. Brenti, Hilbert polynomials in combinatorics. J. Algebraic Combin., 7 (1998), $127-156$.

[20] F. Brenti, Unimodal, log-concave and Pólya frequency sequences in combinatorics. Mem. Amer. Math. Soc., 81 (1989), no. 413.

[21] R. Cori, B. Jacquard, G. Schaeffer, Description trees for some families of planar maps, Proceedings of the 9th Conference on Formal Power Series and Algebraic Combinatorics, (Vienna, 1997), 196-208.

[22] S. Dulucq. S. Gire, J. West, Permutations with forbidden subsequences and nonseparable planar maps. Proceedings of the 5th Conference on Formal Power Series and Algebraic Combinatorics (Florence, 1993), Discrete Math, 153 (1996), 85-103.

[23] P. Edelman, V. Reiner, $h$-shellings and $h$-complexes. Adv. Math., 106 (1994), no. 1, $36-64$.

[24] S. Even, A. Itai, Queues, stacks and graphs, in Z. Kohavi and A. Paz, eds., Theory of Machines and Computations, Proc. Internat. Symp. on the Theory of Machines and Computations, Technion - Israel Inst. of Technol., Haifa, Israel, August 1971 (Academic Press, New York, 1971), 71-86. 
[25] V. Gasharov, On the Neggers-Stanley conjecture and the Eulerian polynomials, J. Combin. Theory Ser. A, 82 (1998), 134-146.

[26] I. P. Goulden, J. West, Raney paths and a combinatorial relationship between rooted nonseparable planar maps and two-stack-sortable permutations, J. Combin. Theory Ser. A, 75 (1996), 220-242.

[27] O. Guibert, Stack words, standard Young tableaux, permutations with forbidden subsequences and planar maps. Formal power series and algebraic combinatorics (Minneapolis, MN, 1996). Discrete Math., 210 (2000), no. 1-3, 71-85.

[28] O. Guibert, A combinatorial proof of J. West's conjecture. Discrete Math., 187 (1998), no. 1-3, 71-96.

[29] D. E. Knuth, "The art of computer programming," volume 1, Fundamental Algorithms, Addison-Wesley, Reading, Massachusetts, 1973.

[30] G. Kreweras, Sur une classe des problèmes liés au treillis des partitions d'entiers. Cahiers du B.U.R.O, 6 (1965), 5-105.

[31] B. Jacquard, G. Schaeffer, A bijective census of nonseparable planar maps. J. Combin. Theory Ser. A, 83 (1998), no. 1, 1-20.

[32] M. M. Murphy, Restricted permutations, antichains, atomic classes and stack sorting, PhD thesis, University of St Andrews, 2002.

[33] V.R. Pratt, Computing permutations with double-ended queues, parallel stacks and parallel queues, Proc. ACM Symp. Theory of Computing, 5 (1973), 268-277

[34] Z. Stankova, J. West, A new class of Wilf-equivalent permutations. J. Algebraic Combin., 15 (2002), no. 3, 271-290.

[35] R. P. Stanley, Log-concave and unimodal sequences in algebra, combinatorics, and geometry, in "Graph Theory and Its Applications: East and West," Ann. NY Acad. Sci. , 576 (1989), 500-535.

[36] R.E. Tarjan, Sorting using networks of queues and stacks, Journal of the ACM, 19 (1972), 341-346.

[37] J. W. Tutte, A census of planar maps, Canadian Journal of Mathematics, 33 (1963), 249-271.

[38] W. Unger, The Complexity of Colouring Circle Graphs STACS'92. Proceedings., A. Finkel, Jantzen, M. (eds.), Springer, Lecture Notes in Computer Science, 5771992 , 389-400.

[39] J. West, Permutations with forbidden subsequences and Stack sortable permutations, PhD-thesis, Massachusetts Institute of Technology, 1990. 
[40] D. Zeilberger, A proof of Julian West's conjecture that the number of two-stacksortable permutations of length $n$ is $2(3 n) ! /((n+1) !(2 n+1) !)$, Discrete Math., 102 (1992), 85-93. 